EPJ Web of Conferences 112,02010 (2016)

DOI: $10.1051 /$ epjconf/201611202010

C) Owned by the authors, published by EDP Sciences, 2016

\title{
Leading neutron production at HERA in the color dipole ap- proach
}

\author{
F. Carvalho ${ }^{1,3, a}$, V.P. Gonçalves ${ }^{2, b}$, D. Spiering ${ }^{3, c}$, and F.S. Navarra ${ }^{3, d}$ \\ ${ }^{1}$ Departamento de Ciências Exatas e da Terra, Universidade Federal de São Paulo, \\ Campus Diadema, Rua Prof. Artur Riedel, 275, Jd. Eldorado, 09972-270, Diadema, SP, Brazil. \\ ${ }^{2}$ High and Medium Energy Group, Instituto de Física e Matemática, Universidade Federal de Pelotas \\ Caixa Postal 354, 96010-900, Pelotas, RS, Brazil. \\ ${ }^{3}$ Instituto de Física, Universidade de São Paulo, \\ C.P. 66318, 05315-970 São Paulo, SP, Brazil.
}

\begin{abstract}
.
In this work we study leading neutron production in $e+p \rightarrow e+n+X$ collisions at high energies and calculate the Feynman $x_{L}$ distribution of these neutrons. The differential cross section is written in terms of the pion flux and of the photon-pion total cross section. We describe this process using the color dipole formalism and, assuming the validity of the additive quark model, we relate the dipole-pion with the well determined dipoleproton cross section. In this formalism we can estimate the impact of the QCD dynamics at high energies as well as the contribution of gluon saturation effects to leading neutron production. With the parameters constrained by other phenomenological information, we are able to reproduce the basic features of the recently released H1 leading neutron spectra.
\end{abstract}

\section{Introduction}

Leading neutron production in ep collisions has been studied for many years [1-8]. Monte Carlo studies, using standard deep inelastic scattering (DIS) generators have convincingly shown [9, 10] that in order to fit the data we need to include pion exchange and even the so-called sub-leading contributions, such as reggeon exchange, baryon resonance excitation (and decay) and independent fragmentation [4-8]. As it can be seen in Fig. 1, this process is composed by a pion (or reggeon) emission and by the subsequent photon-pion interaction at high energies. Since the incoming photon interacts with the pion emitted by the proton and then rescatters, interacting also with the emerging neutron we must include absorptive corrections [5-8]. Moreover the corresponding pion-nucleon

\footnotetext{
ae-mail: babi.usp@gmail.com

be-mail: victorpbg@gmail.com

ce-mail: diego.spiering@gmail.com

$\mathrm{d}_{\mathrm{e}-\mathrm{mail}}$ : navarra@if.usp.br
} 


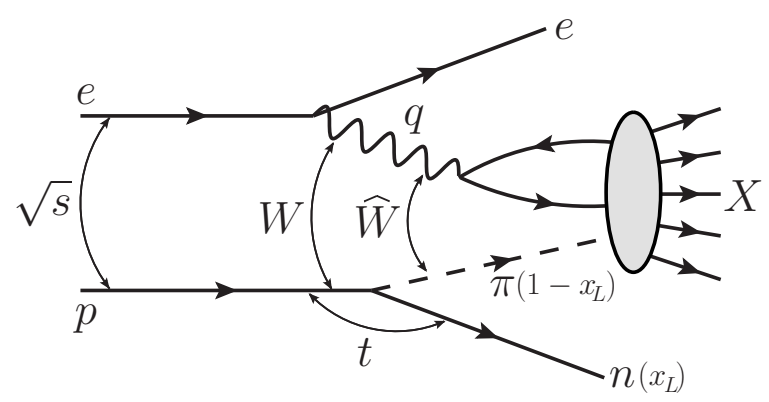

Figure 1. Leading neutron production in the color dipole approach.

vertex must be supplemented with a form factor, which represents the extended nature of hadrons and at the same time regularizes divergent integrals.

An interesting question is the existence or non-existence of the Feynman scaling, which says that the large $x_{L}$ spectra of secondaries are energy independent. In this kinematical region non-linear effects (due to the high parton density) are expected to change the QCD dynamics. The most appropriate framework to treat QCD at high energies is the Color Glass Condensate (CGC) formalism [11], which predicts gluon saturation at small- $x$,

In [12] we have extended the color dipole formalism to the study of leading neutron production in $e+p \rightarrow e+n+X$ collisions at high energies. We have calculated the Feynman $x_{F}$ distribution of leading neutrons. We have considered different models for the dipole scattering amplitude and different forms of the pion flux. We have shown that the recently released H1 leading neutron spectra could be well described using the color dipole formalism and that these spectra could help us to observe more clearly gluon saturation effects in future $e p$ colliders. In that work we could also investigate the relation between Feynman scaling (or its violation) and the high energy QCD dynamics. In the near future, Feynman scaling will be investigated experimentally at the LHC by the LHCf Collaboration, as discussed in [12]. In a subsequent paper [13] with the same formalism we calculated the leading neutron spectrum in the exclusive reaction $e p \rightarrow \rho \pi n$. In [12] we observed that the inclusive data seemed to prefer one particular form of the pion flux. Later, in the analysis performed in [13], the exclusive measurements seemed to prefer another pion flux. In this note, we revisit the fits made in [12], combining them with the results of [13], and try to make a new fit of the inclusive spectra.

\section{Formalism}

Th leading neutron production differential cross section reads:

$$
\frac{d^{2} \sigma\left(W, Q^{2}, x_{L}, t\right)}{d x_{L} d t}=f_{\pi / p}\left(x_{L}, t\right) \sigma_{\gamma^{*} \pi}\left(\hat{W}^{2}, Q^{2}\right)
$$

where $Q^{2}$ is the virtuality of the exchanged photon and $\hat{W}$ is the center-of-mass energy of the virtual photon-pion system. It can be written as $\hat{W}^{2}=\left(1-x_{L}\right) W^{2}$, where $W$ is the center-of-mass energy of the virtual photon-proton system. As it can be seen in Fig. 1, $x_{L}$ is the proton momentum fraction carried by the neutron and $t$ is the square of the four-momentum of the exchanged pion. The flux of virtual pions emitted by the proton is represented by $f_{\pi / p}$ and $\sigma_{\gamma^{*} \pi}\left(\hat{W}^{2}, Q^{2}\right)$ is the cross section of the interaction between the virtual-photon and the virtual-pion at center-of-mass energy $\hat{W}$. 
The pion flux $f_{\pi / p}\left(x_{L}, t\right)$ (also called sometimes pion splitting function) is the virtual pion momentum distribution in a physical nucleon (the bare nucleon plus the "pion cloud"). It has been calculated in different ways by several authors [14]. In all the calculations of the pion flux a form factor was introduced to represent the non-pointlike nature of hadrons and hadronic vertices, which contain a cut-off parameter determined by fitting data. The most frequently used parametrizations of the pion flux [1-8] have the following general form:

$$
f_{\pi / p}\left(x_{L}, t\right)=\frac{1}{4 \pi} \frac{2 g_{p \pi p}^{2}}{4 \pi} \frac{-t}{\left(t-m_{\pi}^{2}\right)^{2}}\left(1-x_{L}\right)^{1-2 \alpha(t)}\left[F\left(x_{L}, t\right)\right]^{2}
$$

where $g_{p \pi p}^{2} /(4 \pi)=14.4$ is the $\pi^{0} p p$ coupling constant, $m_{\pi}$ is the pion mass and $\alpha(t)$ will be defined below. The form factor $F\left(x_{L}, t\right)$ accounts for the finite size of the nucleon and pion. We will consider the following parametrizations of the form factor:

$$
F_{1}\left(x_{L}, t\right)=\exp \left[b\left(t-m_{\pi}^{2}\right)\right], \alpha(t)=\alpha(t)_{\pi}
$$

from Ref. [1], where $b=0.3 \mathrm{GeV}^{-2}$ and

$$
F_{2}\left(x_{L}, t\right)=1, \alpha(t)=\alpha(t)_{\pi}
$$

from Ref. [2], where $\alpha_{\pi}(t) \simeq t$ (with $t$ in $\mathrm{GeV}^{2}$ ) is the Regge trajectory of the pion. In what follows, we shall use the two pion fluxes above denoting them by $f_{1}$ (calculated with (3)) and $f_{2}$ (calculated with (4) ). We will include the absorptive corrections introducing a constant factor $K$ which multiplies the uncorrected cross section of leading neutron production.

In order to obtain the photon-pion cross section we will use the color dipole formalism, as usually done in high energy deep inelastic scattering off a nucleon target. In this formalism, the cross section is factorized in terms of the photon wave functions $\Psi$, which describes the photon splitting in a $q \bar{q}$ pair, and the dipole-pion cross section $\sigma_{d \pi}$. It reads:

$$
\sigma_{\gamma^{*} \pi}\left(\hat{x}, Q^{2}\right)=\int_{0}^{1} d z \int d^{2} \boldsymbol{r} \sum_{L, T}\left|\Psi_{T, L}\left(z, \boldsymbol{r}, Q^{2}\right)\right|^{2} \sigma_{d \pi}(\hat{x}, \boldsymbol{r})
$$

where $\hat{x}=\left[Q^{2}+m_{f}^{2}\right] /\left[\left(1-x_{L}\right) W^{2}+Q^{2}\right]$ is the scaled Bjorken variable and the variable $\boldsymbol{r}$ defines the relative transverse separation of the pair (dipole). The photon wave function is easy to compute in QED and its expression can be found in textbooks [15]. As usual $z$ stands for the longitudinal photon momentum fraction carried by the quark and $1-z$ is the longitudinal photon momentum fraction of the antiquark.

The main input in the calculations of $\sigma_{\gamma^{*} \pi}$ is the dipole-pion cross section. In what follows, for simplicity, we will assume the validity of the additive quark model, which allows us to relate $\sigma_{d \pi}$ with the dipole-proton cross section, usually probed in the typical inclusive and exclusive processes at HERA. Basically, we will assume that

$$
\sigma_{d \pi}(x, \boldsymbol{r})=R_{q} \cdot \sigma_{d p}(x, \boldsymbol{r})
$$

where $R_{q}=2 / 3$ is the ratio between the number of valence quarks in the hadrons. This assumption is supported by the study of the pion structure function in the low $x$ regime presented in [16]. It also gives a good description of the previous ZEUS leading neutron spectra, as shown in [5, 6]. On the other hand, the direct application of (1) to HERA photoproduction data [17] leads to the result $\sigma_{\gamma \pi}^{\mathrm{tot}} / \sigma_{\gamma p}^{\mathrm{tot}}=0.32 \pm 0.03$, which is factor 2 lower than the ratio given above. The effective value of $R_{q}$ 
was more recently discussed in [18] on more theoretical grounds with the conclusion that this number could reach the value of $R_{q}=0.5$. We shall use relation (6) to estimate $\sigma_{d \pi}$ letting $R_{q}$ vary from $1 / 3$ to $2 / 3$.

In the eikonal approximation the dipole-proton cross section $\sigma_{d p}$ is given by:

$$
\sigma_{d p}(x, \boldsymbol{r})=2 \int d^{2} \boldsymbol{b} \mathcal{N}^{p}(x, \boldsymbol{r}, \boldsymbol{b})
$$

where $\mathcal{N}^{p}(x, \boldsymbol{r}, \boldsymbol{b})$ is the imaginary part of the forward amplitude for the scattering between a dipole with transverse size given by the vector $\boldsymbol{r}=\boldsymbol{x}-\boldsymbol{y}$, where $\boldsymbol{x}$ and $\boldsymbol{y}$ are the transverse vectors for the quark and antiquark, respectively, and impact parameter $\boldsymbol{b}=(\boldsymbol{x}+\boldsymbol{y}) / 2$. Assuming the translational invariance approximation, which implies $\mathcal{N}^{p}(x, \boldsymbol{r}, \boldsymbol{b})=\mathcal{N}^{p}(x, \boldsymbol{r}) S(\boldsymbol{b})$ and $\sigma_{d p}(x, \boldsymbol{r})=\sigma_{0} \cdot \mathcal{N}^{p}(x, \boldsymbol{r})$, with the normalization of the dipole cross section, $\sigma_{0}$, being fitted to data. In what follows we will use as input the phenomenological model proposed in Ref. [19]. In this parametrization the forward dipole-proton scattering amplitude is given by:

$$
\mathcal{N}^{p}(x, \boldsymbol{r})= \begin{cases}\mathcal{N}_{0}\left(\frac{r Q_{s}}{2}\right)^{2\left(\gamma_{s}+\frac{\ln \left(2 / r Q_{s}\right)}{\kappa \lambda Y}\right)}, & \text { for } r Q_{s}(x) \leq 2, \\ 1-\mathrm{e}^{-a \ln ^{2}\left(b r Q_{s}\right)}, & \text { for } r Q_{s}(x)>2,\end{cases}
$$

where $a$ and $b$ are determined by continuity conditions at $r Q_{s}(x)=2, \gamma_{s}=0.7376, \kappa=9.9, \mathcal{N}_{0}=0.7$ and $Q_{s}$ is the saturation scale given by $Q_{s}^{2}(x)=Q_{0}^{2}\left(x_{0} / x\right)^{\lambda}$ with $x_{0}=1.632 \times 10^{-5}, \lambda=0.2197$, $Q_{0}^{2}=1.0 \mathrm{GeV}^{2}$. We will also use the GBW model [20], which captures the basic features of saturation physics and allows for simple analytical estimates. The GBW dipole-proton scattering amplitude is given by:

$$
\mathcal{N}^{p}(x, \boldsymbol{r})=1-\exp \left[-\frac{Q_{s}^{2} r^{2}}{4}\right]
$$

with $Q_{0}^{2}=1.0 \mathrm{GeV}^{2}, x_{0}=3 \times 10^{-4}$ and $\lambda=0.288$. Finally, we will also use the dipole-proton cross section estimated with the help of the DGLAP analysis of the gluon distribution, which is given by [15]:

$$
\sigma_{\text {dip }}(x, \boldsymbol{r})=\frac{\pi^{2}}{3} r^{2} \alpha_{s} x g\left(x, 10 / r^{2}\right)
$$

where $x g\left(x, Q^{2}\right)$ is the target gluon distribution, for which we use the CTEQ6 parametrization [21]. The above expression represents the linear regime of QCD and is a baseline for comparison with the non linear predictions.

\section{Results and discussion}

Using the two parametrizations of the pion flux, $f_{1}$ and $f_{2}$, introduced in the previous section to compute the leading neutron spectra, which are shown in In Fig. 2. The normalization of the spectrum was taken from an experimental analysis, as explained in [12]. The $f_{2}$ model has a long and comparatively flat tail in the low $x_{L}$ region, which makes possible a fit of the data in this region even without including a contribution from other processes. The $f_{1}$ model falls too quickly at low $x_{L}$. However, the sub-leading contributions mentioned in [12] are more important in the low $x_{L}$ region of the spectrum and their inclusion combined with $f_{1}$ might result in a good fit of the data as well.

In what follows we shall include the effect of absorptive corrections multiplying our spectra by a constant (independent of $x_{L}$ ) factor $K$ which can assume values from 0.5 to 1 . In Fig. 3 we show our results for the leading neutron spectra. The bands account for the uncertainty in the choice of 


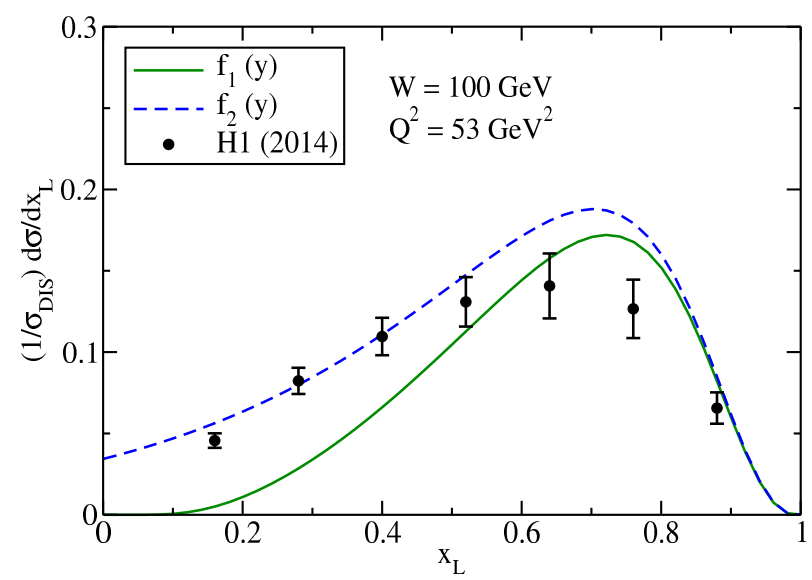

Figure 2. Leading neutron spectra compared with experimental data at $W=100 \mathrm{GeV}$ considering the IIMS scattering amplitude and different choices of the pion flux $f_{1}$ and $f_{2}$.

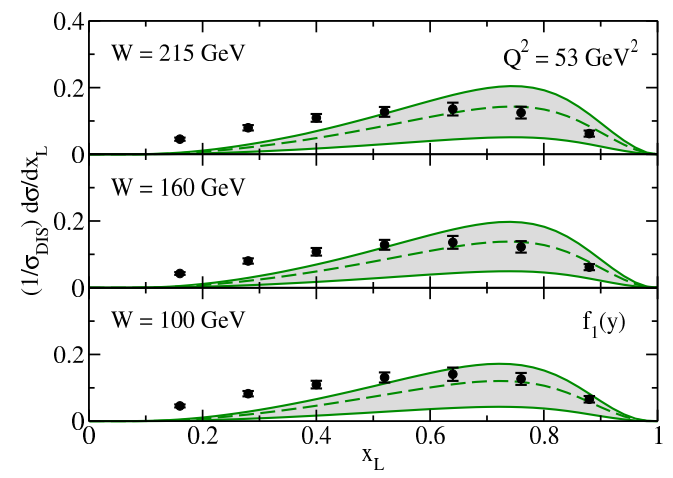

(a)

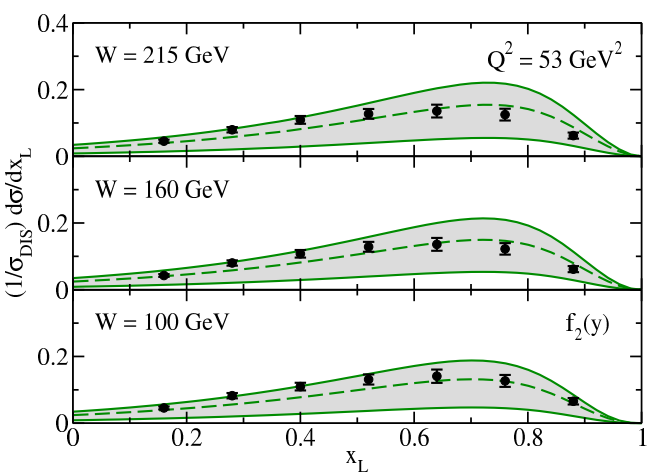

(b)

Figure 3. Leading neutron $x_{L}$ spectrum for three photon-proton energies compared with recent H1 data. a) Calculated with $f_{1}$. b) Calculated with $f_{2}$. The bands are explained in the text.

the factors $R_{q}$ and $K$. The upper limit of each band corresponds to the choice $R_{q}=2 / 3$ and $K=1$ whereas the lower limit corresponds to the choice $R_{q}=1 / 3$ and $K=0.5$. The central dashed lines represent the choice where $R_{q} \cdot K=0.5$, which could, for example, be realized with $R_{q}=0.5$ and $K=1$. Our results follow the trend dictated by data. For now, it is not possible to be very precise. With a combined analysis of other data on exclusive production with tagged leading neutrons we hope to reduce the bands.

We now address the Feynman scaling in leading neutron spectra and the contribution of non linear effects to this process. The theoretical expectation can be obtained using the GBW model for the scattering amplitude, Eq. (9). In the linear limit, when the dipole radius is very small (or equivalently $Q^{2}$ is very large) or the saturation scale is very small (and hence the energy is not very high), we can 


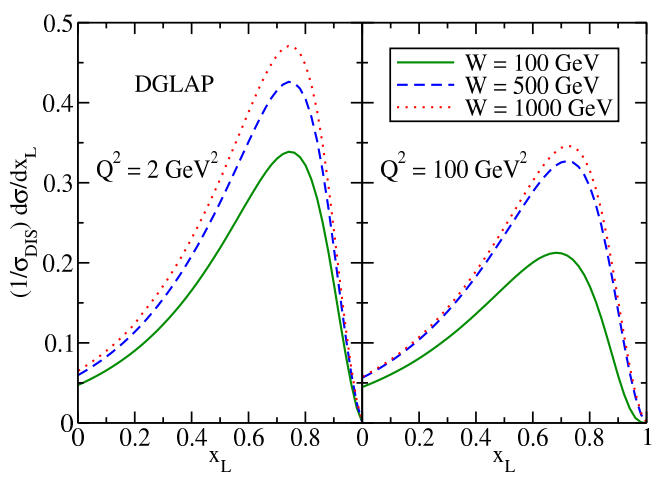

(a)

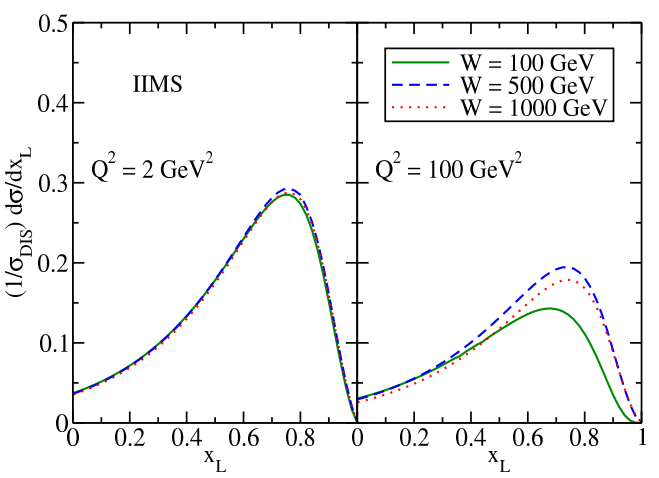

(b)

Figure 4. Leading neutron spectra for different energies considering (a) the linear DGLAP model and (b) the nonlinear IIMS model for the dipole-pion cross section.

expand the exponent and obtain

$$
\sigma_{d \pi}(r, \hat{x}) \propto \sigma_{0} \mathcal{N}(r, \hat{x}) \simeq \sigma_{0} \frac{Q_{s}^{2}(\hat{x}) r^{2}}{4} \simeq \sigma_{0} Q_{0}^{2} x_{0}{ }^{\lambda}\left[\frac{\left(1-x_{L}\right) W^{2}+Q^{2}}{Q^{2}+m_{f}^{2}}\right]^{\lambda}
$$

Consequently, in this regime we see that the leading neutron $x_{L}$ spectrum will depend on $W$. In a complementary way, in the nonlinear limit, when the dipole radius is very large (or equivalently $Q^{2}$ is very small) or the saturation scale is very large (and hence the energy is very high), we obtain

$$
\sigma_{d \pi}(r, \hat{x}) \propto \sigma_{0} \mathcal{N}(r, \hat{x}) \simeq \sigma_{0}
$$

which is energy independent. The information contained in (11) and (12) indicates the route through which the asymptotic limit is reached and the role played by non linear effects. These expectations can be compared with those obtained using the IIMS and DGLAP models for the dipole-pion cross section. In Fig. 4(a) we show the spectra obtained in a purely linear approach. As expected we see a noticeable energy dependence. In contrast, the non linear predictions presented in Fig. 4(b) show a remarkable suppression of the energy dependence at low values of $Q^{2}$, consistent with the expectations. These results indicate that the Feynman scaling (and how it is violated) can be directly related to the QCD dynamics at small- $x$. As it was shown in [13], it is possible to constrain the unknown numbers and assumptions with the help of more experimental data on exclusive processes with tagged leading neutrons. Our most interesting conclusion is that leading neutron spectra can be used to probe the low $x$ content of the pion target and hence it is a new observable where we can look for gluon saturation effects.

\section{References}

[1] B. Kopeliovich, B. Povh and I. Potashnikova, Z. Phys. C 73, 125 (1996).

[2] M. Bishari, Phys. Lett. B 38, 510 (1972). 
[3] H. Holtmann et al., Phys. Lett. B 338, 363 (1994); S. Kumano, Phys. Rev. D 43, 59 (1991); N.N. Nikolaev, W. Schaefer, A. Szczurek and J. Speth, Phys. Rev. D 60, 014004 (1999).

[4] L.L. Frankfurt, L. Mankiewicz and M.I. Strikman, Z. Phys. A 334, 343 (1989); T.T. Chou and C.N. Yang, Phys. Rev. D 50, 590 (1994); K. Golec-Biernat, J. Kwiecinski and A. Szczurek, Phys. Rev. D 56, 3955 (1997); M. Przybycien, A. Szczurek and G. Ingelman, Z. Phys. C 74, 509 (1997); W. Melnitchouk, J. Speth and A.W. Thomas, Phys. Rev. D 59, 014033 (1998); A. Szczurek, N.N. Nikolaev and J. Speth, Phys. Lett. B 428, 383 (1998).

[5] A.B. Kaidalov,V.A. Khoze, A.D. Martin and M.G. Ryskin, Eur. Phys. J. C 47, 385 (2006).

[6] V.A. Khoze, A.D. Martin and M.G. Ryskin, Eur. Phys. J. C 48, 797 (2006).

[7] N.N. Nikolaev, J. Speth and B.G. Zakharov, hep-ph/9708290.

[8] U. D' Alesio and H.J. Pirner, Eur. Phys. J. A 7, 109 (2000).

[9] J. Olsson [H1 Collaboration], PoS DIS 2014, 156 (2014); V. Andreev et al. [H1 Collaboration], Eur. Phys. J. C 74, 2915 (2014); F. D. Aaron et al. [H1 Collaboration], Eur. Phys. J. C 68, 381 (2010).

[10] See, for example, ZEUS Collaboration, M. Derrick et al., Phys. Lett. B 384, 388 (1996).

[11] J. L. Albacete and C. Marquet, Prog. Part. Nucl. Phys. 76, 1 (2014) and referecnes therein.

[12] F. Carvalho, V. P. Gonçalves, D. Spiering and F. S. Navarra, Phys. Lett. B 752, 76 (2016).

[13] V. P. Gonçalves, F. S. Navarra and D. Spiering, arXiv:1512.06594 [hep-ph].

[14] J.D. Sullivan, Phys. Rev. D 5, 1732 (1972); A.W. Thomas, Phys. Lett. B 126, 97 (1983); J. Speth and A. W. Thomas, Adv. Nucl. Phys. 24, 83 (1998); S. Kumano, Phys. Rep. 303, 183 (1998); F. S. Navarra, M. Nielsen, C. A. A. Nunes and M. Teixeira, Phys. Rev. D 54, 842 (1996); S. Paiva, M. Nielsen, F. S. Navarra, F. O. Durães and L. L. Barz, Mod. Phys. Lett. A 13, 2715 (1998); W. Melnitchouk and A.W. Thomas, Phys. Lett. B 414, 134 (1997); F. Carvalho, F. O. Duraes, F. S. Navarra, M. Nielsen and F. M. Steffens, Eur. Phys. J. C 18, 127 (2000).

[15] V. Barone and E. Predazzi, High-Energy Particle Diffraction, Springer-Verlag, Berlin Heidelberg, (2002).

[16] N.N. Nikolaev, J. Speth and V.R. Zoller, Phys. Lett. B 473, 157 (2000).

[17] S. Chekanov et al. [ZEUS Collaboration], Nucl. Phys. B 637, 3 (2002).

[18] B. Z. Kopeliovich, I. K. Potashnikova, B. Povh and I. Schmidt, Phys. Rev. D 85, 114025 (2012).

[19] G. Soyez, Phys. Lett. B 655, 32 (2007).

[20] K. J. Golec-Biernat, M. Wusthoff, Phys. Rev. D 59, 014017 (1998).

[21] H. L. Lai, J. Huston, Z. Li, P. Nadolsky, J. Pumplin, D. Stump and C.-P. Yuan, Phys. Rev. D 82, 054021 (2010). 